IOS Press

\title{
Erratum
}

\section{Cerebral Microbleeds Are Associated with Cerebral Hypoperfusion in Patients with Alzheimer's Disease}

Yutaka Hatada, Mamoru Hashimoto, Shinya Shiraishi, Tomohisa Ishikawa, Ryuji Fukuhara, Seiji Yuki, Hibiki Tanaka, Yusuke Miyagawa, Mika Kitajima, Hiroyuki Uetani, Naoko Tsunoda, Asuka Koyama and Manabu Ikeda

[Journal of Alzheimer's Disease, 71(1) 2019, 272-280, DOI 10.3233/JAD-190272]

https://content.iospress.com/articles/journal-of-alzheimers-disease/jad190272

on page 273, the following affiliation is given for Yutaka Hatada:

"a Department of Psychiatry, Heisei Hospital, Yatsushiro, Kumamoto, Japan"

However, this should be corrected to:

"a Department of Psychiatry, Heisei Hospital, Yatsushiro, Kumamoto, Japan" and "d Department of Neuropsychiatry, Kumamoto University Hospital, Kumamoto, Japan"

On page 279, in the Acknowledgement section, the authors gave the following acknowledgement:

"The authors gratefully acknowledge the assistance of the Department of Radiology and the Department of Neuropsychiatry, Kumamoto University Hospital."

They would like to change this to:

"The authors received useful advice from Professor Minoru Takebayashi, Department of Neuropsychiatry, Kumamoto University Hospital. And we gratefully acknowledge the assistance of the Department of Radiology and the Department of Neuropsychiatry, Kumamoto University Hospital." 